\title{
Stellar and interstellar parameters from large photometric surveys
}

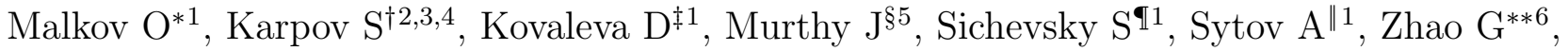 \\ Zhukov $\mathrm{A}^{\dagger \dagger 1,7,8}$, and Zakharov $\mathrm{A}^{\ddagger \ddagger 9}$ \\ ${ }^{1}$ Institute of Astronomy, Moscow 119017, Russia \\ ${ }^{2}$ Institute of Physics, Czech Academy of Sciences, 18221 Prague 8, Czech Republic \\ ${ }^{3}$ Special Astrophysical Observatory, Nizhnij Arkhyz 36916, Russia \\ ${ }^{4}$ Kazan Federal University, Kazan 420008, Russia \\ ${ }^{5}$ Indian Institute of Astrophysics, Bengaluru 560034, India \\ ${ }^{6}$ National Astronomical Observatories, Beijing 100012, China \\ 7 "MPEI Special Design Bureau" Corporation, Moscow, Russia \\ ${ }^{8}$ Federal State Budgetary Scientific Institution "Expert and Analytical Center", Moscow, Russia \\ ${ }^{9}$ Sternberg Astronomical Institute, Moscow, Russia
}

\begin{abstract}
The parameterization of stars is a well known problem and used for various purposes in astronomy. We have shown that multicolor photometric data from large modern surveys can be used for parameterization of stars. With sufficiently good quality photometry, one may compute a 3D extinction map by comparing catalogued multicolor photometry with photometry derived from the secondary estimators such as the distance modulus and the interstellar extinction law with suitable calibration tables for absolute magnitudes with reasonable spectral types, extinctions and distances.
\end{abstract}

Keywords: Sky surveys $\cdot$ Photometry $\cdot$ Cross-matching $\cdot$ Interstellar extinction

\section{Introduction}

Three-dimensional (3D) extinction models have been constructed using spectral and photometric stellar data, open cluster data, star counts, Galactic dust distribution models. The standard approach to construct a 3D extinction model has been to parcel out the sky in angular cells, each defined by boundaries in Galactic coordinates $(1, \mathrm{~b})$. The visual extinction $\left(A_{V}\right)$ in each cell may then be obtained as a function of distance (d): $A_{V}(\mathrm{l}, \mathrm{b}, \mathrm{d})$ from the stars in the cells. The angular size of the cells has varied from study to study, although each cell was generally chosen to be large enough to contain a statistically significant number of calibration stars at different distances.

Published 3D models, using spectral and photometric data, were based on $10^{4}-10^{5}$ stars, or were constructed for a very limited area in the sky (see, e.g., Sale et al. (2014), Green \& et al (2015), Lallement et al. (2018), the earlier studies were reviewed in Malkov \& Kilpio (2002)). Modern large surveys contain photometric ( 3 to 5 bands) data for $10^{7}-10^{9}$ stars. However, to make those data (obtained at different wavelengths and with different observational techniques) useful for a 3D extinction model construction, one needs to run a correct cross-identification of objects between surveys. Such crossidentification was laborious and time consuming, but using Virtual Observatory (VO) data access and

\footnotetext{
*malkov@inasan.ru, Corresponding author

${ }^{\dagger}$ karpov.sv@gmail.com

‡dana@inasan.ru

§murthy@iiap.res.in

$\mathbb{I}_{\text {s.sichevskij@gmail.com }}$

॥ sytov@inasan.ru

** gzhao@nao.cas.cn

††aozhukov@mail.ru

¥¥zakhster@gmail.com
} 
cross-correlation technologies, a search for counterparts in a subset of different catalogues can now be carried out in a few minutes. It is now feasible to obtain information on interstellar extinction from modern large photometric surveys.

In Section 2 we give a review of sky surveys, and present principles of their cross-matching. Section 3 contains description of our procedure for parameterization of stars, and in Section 4 we present principles and pilot results of determination of interstellar extinction. We draw our conclusions in Section 5 .

\section{Sky Surveys and Cross-matching}

\subsection{Sky Surveys Selection}

The number of surveys available at any wavelength is large enough to construct detailed spectral energy distributions (SED) for any kind of astrophysical object. Sky surveys, we select for parameterization of stars, should satisfy the following criteria:

- the number of objects exceeds $\sim 10^{7}$,

- the survey covers a large area in the sky,

- the photometric accuracy is better than about $0.05 \mathrm{mag}$,

- the depth of the survey exceeds $\mathrm{V} \sim 20 \mathrm{mag}$.

Table 1. Large photometric surveys

\begin{tabular}{|c|c|c|c|c|}
\hline Survey & $\mathrm{N}^{\mathrm{a}}$ & $\begin{array}{l}\text { Sky } \\
\text { coverage }\end{array}$ & $\begin{array}{l}\text { Photometric } \\
\text { bands }\end{array}$ & $\begin{array}{l}\text { Limiting } \\
\text { magnitude }\end{array}$ \\
\hline DENIS & 355 & $\begin{array}{l}\text { Southern } \\
\text { hemisphere }\end{array}$ & Gunn-i, J, K ${ }_{S}$ & $\begin{array}{l}18.5,16.5, \\
14.0\end{array}$ \\
\hline 2MASS & 471 & All sky & $\mathrm{J}, \mathrm{H}, \mathrm{K}_{S}$ & $\begin{array}{l}15.8,15.1, \\
14.3\end{array}$ \\
\hline SDSS 12 & 325 & $25 \%$ & $\mathrm{u}, \mathrm{g}, \mathrm{r}, \mathrm{i}, \mathrm{z}$ & $\mathrm{g}, \mathrm{r}=22.2$ \\
\hline $\begin{array}{c}\text { GALEX DR5 } \\
\text { (AIS+MIS) }\end{array}$ & 78 & $90 \%$ & FUV, NUV & $\sim 25$ \\
\hline $\begin{array}{l}\text { UKIDSS DR9 } \\
\text { LAS }\end{array}$ & 83 & $15 \%$ & $\mathrm{Z}, \mathrm{Y}, \mathrm{J}, \mathrm{H}, \mathrm{K}$ & $\mathrm{K}=18.3$ \\
\hline AllWISE & 748 & All sky & $\begin{array}{l}\text { W1, W2, } \\
\text { W3, W4 }\end{array}$ & $\begin{array}{l}16.6,16.0 \\
10.8,6.7\end{array}$ \\
\hline IPHAS DR2 & 219 & $\begin{array}{l}\text { Northern } \\
\text { Galactic } \\
\text { plane }\end{array}$ & $\mathrm{r}, \mathrm{i}, H_{\alpha}$ & $\mathrm{r}=21-22$ \\
\hline $\begin{array}{c}\text { Pan-STARRS } \\
\text { PS1 - DR1 }\end{array}$ & 1919 & $\begin{array}{l}\text { All sky but } \\
\text { southern cap }\end{array}$ & $\mathrm{g}, \mathrm{r}, \mathrm{i}, \mathrm{z}, \mathrm{y}$ & $\mathrm{i} \sim 20$ \\
\hline GAIA DR2 & 1693 & All sky & $\mathrm{G}, \mathrm{BP}, \mathrm{RP}$ & $\mathrm{G}=20$ \\
\hline
\end{tabular}

The following sky surveys are selected for our study.

- The DENIS database DENIS Consortium (2005)

- 2MASS All-Sky Catalog of Point Sources Cutri \& et al (2003)

- The SDSS Photometric Catalogue, Release 12 Alam et al. (2015) 


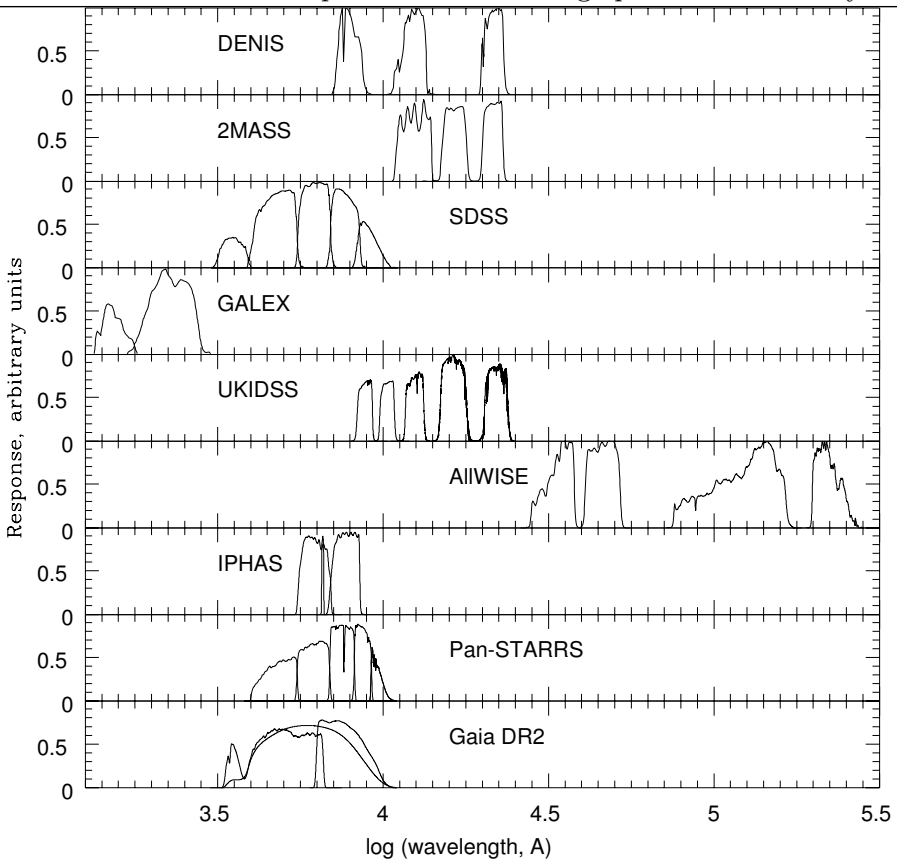

Figure 1. Response curves of the photometric surveys.

- GALEX-DR5 (GR5) sources from AIS and MIS Bianchi et al. (2011), Bianchi et al. (2017)

- UKIDSS-DR9 LAS, GCS and DXS Surveys Lawrence \& et al (2007)

- AllWISE Data Release Cutri \& et al (2014)

- IPHAS DR2 Source Catalogue Barentsen \& et al (2014)

- The Pan-STARRS release 1 (PS1) Survey - DR1 Chambers \& et al (2016)

- Gaia DR2 Gaia Collaboration et al. (2018), Bailer-Jones et al. (2018)

Some information on the surveys is given in Table 1, their photometric systems response curves are shown in Fig. 1.

The presented surveys basically satisfy the criteria listed above. The number of objects vary from $\sim 10^{8}$ to $\sim 10^{9}$. The most local survey, IPHAS, covers a relatively small but important area in the sky. The photometric accuracy of all surveys (except, probably, ultraviolet GALEX) is better than 0.05 mag. The limiting magnitudes, converted to V-band, are not less than $\sim 20$ mag.

\subsection{Cross-matching of Surveys}

Different surveys/instruments have different positional accuracy and resolution. In addition, the depth of each survey is different and, depending on sources brightness and their SED, a given source might or might not be detected at a certain wavelength. All this makes the pairing of sources among surveys not trivial, especially in crowded fields.

We have implemented an algorithm of fast positional matching of some of the sky surveys in small (up to one degree) areas with filtering of false identification Malkov \& Karpov (2011). In particular, for each area and each pair we estimated the matching radius. As a result, we drew in a number 0.1-degree radius areas samples of point-like objects counterparts from the DENIS, 2MASS, SDSS, GALEX, and UKIDSS surveys, and performed a cross-identification within these surveys Karpov et al. (2012), Malkov et al. (2012). We have compiled the corresponding subcatalogues in the VOTable format. The tool developed as a result of this work can be used to cross-identify objects in arbitrary sky areas for the further classification and determination of stellar parameters, including the measurement of the amount of interstellar extinction. 
Stellar and interstellar parameters from large photometric surveys

In some surveys (e.g., GALEX, SDSS, UKIDSS) more than one observation per object was made and, consequently, more than one entry per object is present in the survey. In such cases we use weighted average values for the photometry.

In the cross-identification process (and later for the parameterization) we use all positional information and all photometry available in surveys. To select objects for further study we also pay attention to various flags, presented in the surveys. The flags can indicate quality of observations and provide information on a nature of object (duplicity, variability, extended shape). As it was mentioned above, on this stage we do not use trigonometric parallax as an input parameter.

It can be seen from Fig.1 that response curves of photometric bands in different surveys are sometimes the same or similar (e.g., $K_{S}$-band in DENIS and $K_{S}$-band in 2MASS). The comparison of brightness of objects in such pairs of bands provides us an additional filter to discard objects irrelevant for the parameterization: a large magnitude difference may indicate variability, a rare evolutionary stage, or non-stellar nature of the object.

We have to remove all non-stellar objects, unresolved photometric binaries, variable stars and other contaminating objects, based on flags included in the original surveys. Too bright and too faint objects for this particular survey (i.e., overexposed and underexposed, respectively) can also be spotted and omitted at this stage.

\section{Multicolor Photometry and Parameterization of Stars}

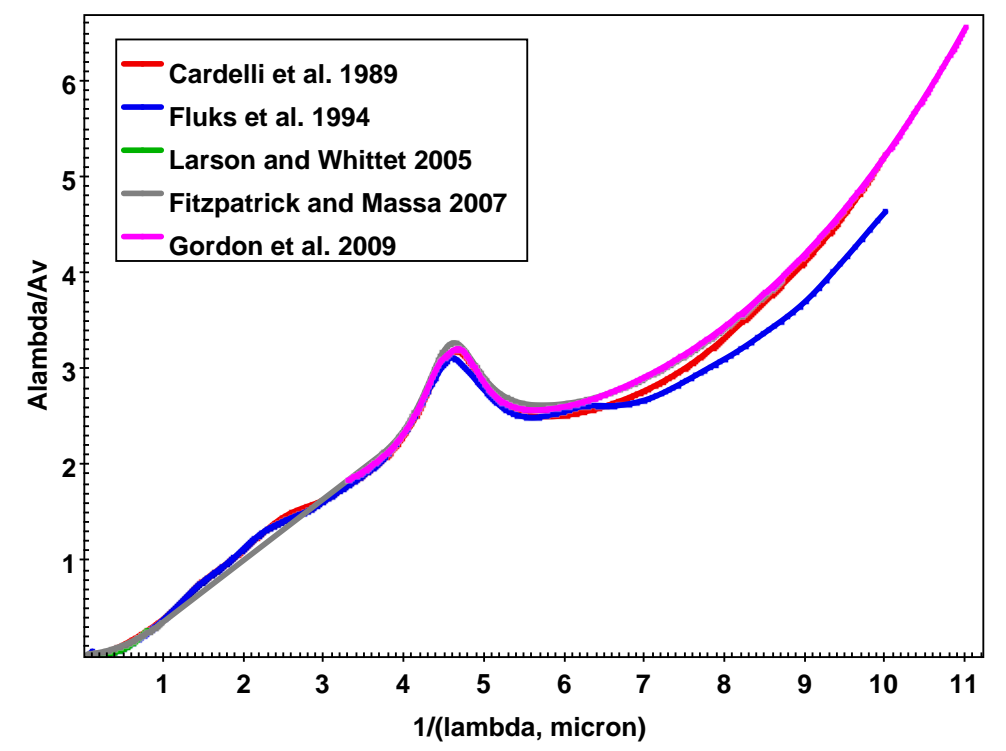

Figure 2. Interstellar extinction law. Red curve: Cardelli et al. (1989) for $R_{V}=3.1$. Blue curve: Fluks et al. (1994) for $R_{V}=3.1$. Green curve: Larson \& Whittet (2005). Grey curve: Fitzpatrick \& Massa (2007) for $R_{V}=3.1$. Purple curve: Gordon et al. (2009) for $R_{V}=3.1$.

We studied a problem of classification and parameterization of stars from multicolor photometry in detail (see, e.g., Sichevskiy et al. (2013), Sichevsky \& Malkov (2016)). In particular, a problem of binary stars parameterization was studied in Malkov et al. (2010) and Malkov et al. (2011).

We have developed a method, which allows us to determine stellar parameters from multicolor photometry. For the studied objects MK (Morgan-Keenan) spectral types (SpT), distances $(d)$ and interstellar extinction values $\left(A_{V}\right)$ can be estimated, minimizing the function

$$
\chi^{2}=\sum_{i=1}^{N}\left(\frac{m_{o b s, i}-m_{c a l c, i}}{\sigma m_{o b s, i}}\right)^{2},
$$

where $m_{o b s, i}$ and $\sigma m_{o b s, i}$ are the apparent magnitude and its observational error, respectively, in the $i$-th photometric band from a given survey. The summation is over up to $\mathrm{N} \sim 30$ photometric bands 


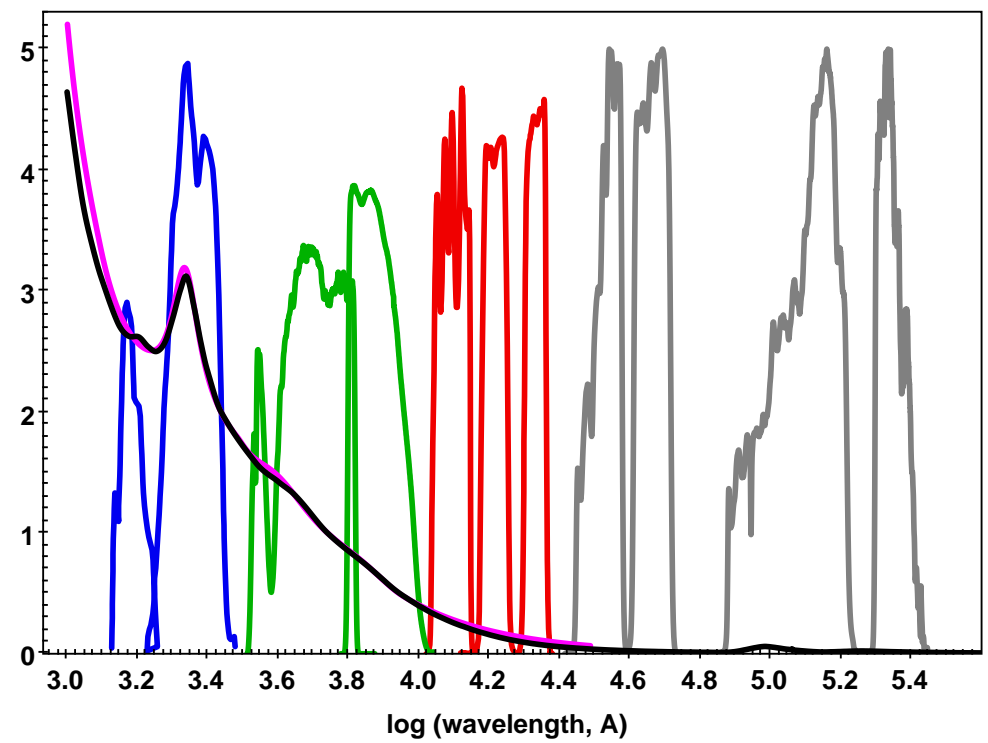

Figure 3. Response curves of all-sky photometric surveys and IEL. Response curves, from left to right: GALEX (FUV, NUV), Gaia (BP, RP), 2MASS (J, H, K ${ }_{S}$ ), AllWISE (W1, W2, W3, W4); the Y-axis represents the capacity in arbitrary units. IELs for $R_{V}=3.1$ : Cardelli et al. (1989) (purple curve), Fluks et al. (1994) (black curve); the Y-axis represents $A_{\lambda} / A_{V}$.

(see Table 1), and

$$
m_{\text {calc }, i}=M_{i}(\mathrm{SpT})+5 \log \mathrm{d}-5+\mathrm{A}_{\mathrm{i}}\left(\mathrm{A}_{\mathrm{V}}\right),
$$

Here $A_{i}=f\left(A_{V}\right)$ is the extinction in the i-th photometric band, and can be determined from the interstellar extinction law (Fluks et al. (1994), Cardelli et al. (1989), Fitzpatrick \& Massa (2007)).

A calibration relation $M_{i}(\mathrm{SpT})$ should be available for each of the $i$ photometric bands included in the original surveys. It is true for 2MASS, SDSS and GALEX photometric systems (Kraus \& Hillenbrand (2007), Findeisen et al. (2011)) but corresponding calibration tables for UKIDSS and other surveys can not be found in literature. In the absence of such information, it is necessary to construct corresponding relations from theoretical spectral energy distributions (SED) and photometric system response curves. The best source for theoretical SED are libraries of synthetic spectra Lejeune et al. (1997), Castelli \& Kurucz (2003), Gustafsson et al. (2008), and the SEDs are computed there for a given set of atmospheric parameters $\left(\log T_{\text {eff }}, \log g,[M / H]\right)$ rather than spectral classes. Thus, for the decision of this problem it is necessary to design and use relations between spectral class and atmospheric parameters, for different luminosity classes. To construct analytic (spectral class - atmospheric parameters) formula, we have used data from the empirical stellar spectral atlases ELODIE (Prugniel et al., 2007), Indo-US (Valdes et al., 2004), MILES (Falcón-Barroso et al., 2011), and STELIB (Le Borgne et al., 2003), and made a polynomial approximation. The results are presented in Table 3 (see Malkov et al. (2020) for details).

Another source of SED for different types of stars (which can be used to model observational photometry), are empirical spectrophotometric atlases (e.g., Pickles (1998), Wu et al. (2011)). We have made a comparative analysis of the most known semi-empirical and empirical spectral atlases. The results show that the standard error of synthesized stellar magnitudes calculated with SED from best spectral atlases reaches $0.02 \mathrm{mag}$. It has been also found that some modern spectral atlases are burdened with significant systematic errors Kilpio et al. (2012).

To parameterize the stars it is necessary to have an understanding of the relation of interstellar extinction in V-band and in photometric bands of the used surveys, $A_{i}\left(A_{V}\right)$ (see Eq. 2). However, $A_{i}\left(A_{V}\right)$ values are calculated and available in the literature only for a small number of modern photometric systems, namely: Rieke \& Lebofsky (1985) (UBVRIJHKLMN, 8.0,8.5,..13.0 $\mu \mathrm{m}$ ), Cardelli et al. (1989) (UBVRIJHKL), Draine (2003) (Johnson, Cousins, SDSS), Indebetouw et al. (2005) (2MASS, Spitzer), Schlafly \& Finkbeiner (2011) (Landolt, UKIRTJHKL, Gunn, Stromgren, SDSS), Yuan et al. (2013) (2MASS, SDSS, GALEX, AllWISE), Hanson \& Bailer-Jones (2014) (u,g,i,z,Y,J,H,K), Bono 
Table 2. Spectral class - effective temperature - surface gravity relations

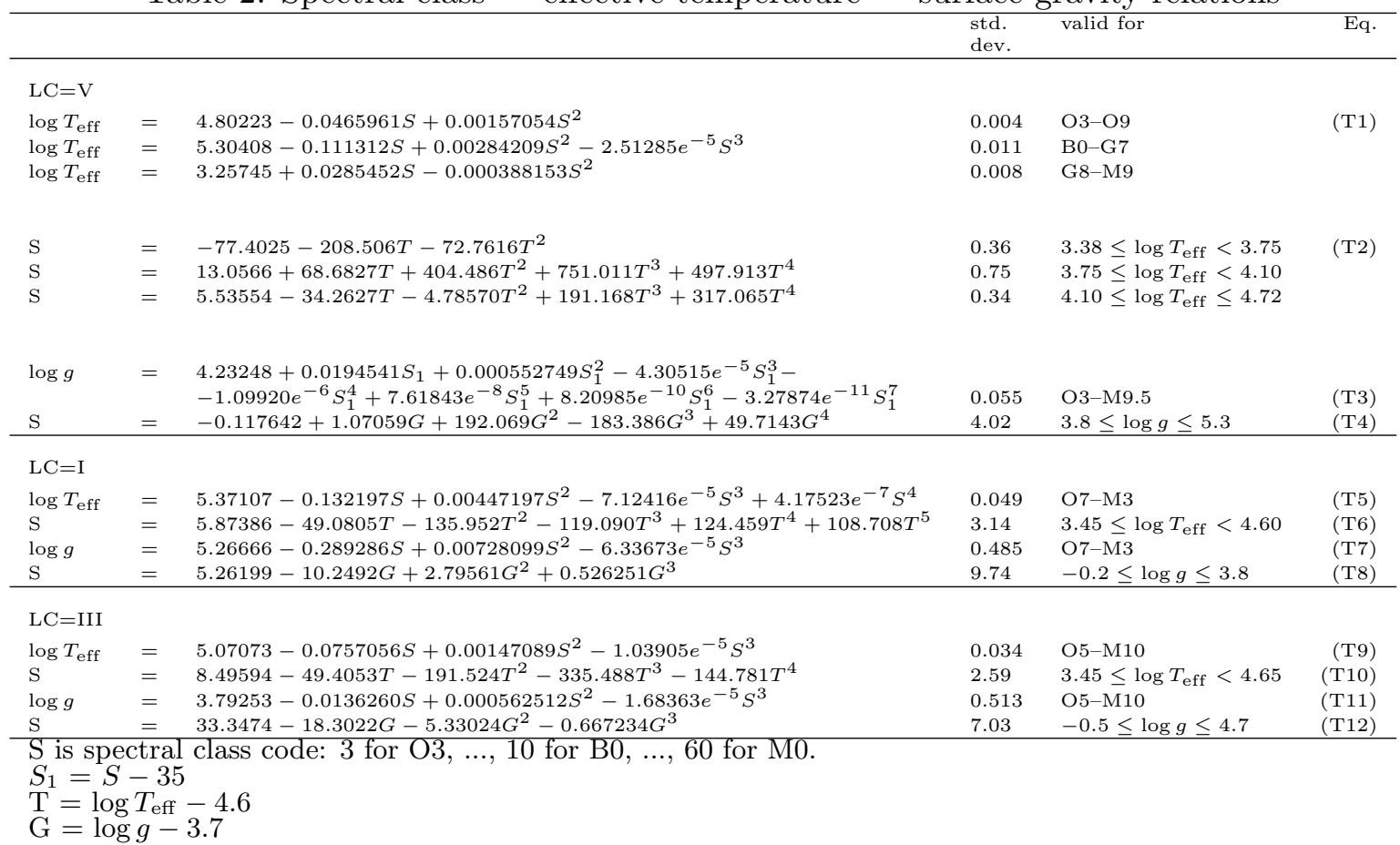

et al. (2019) (Gaia). To calculate the missing $A_{i}\left(A_{V}\right)$ values it is necessary to know the interstellar extinction law, IEL (see a summary of published IELs in Table 3 and Figs. 2 and 3). The value $x \equiv(\lambda, \mu m)^{-1}$ is commonly used as argument in the IEL.

Table 3. Interstellar extinction law

\begin{tabular}{llll}
\hline Reference & $\mathrm{x},(\mu m)^{-1}$ & $\lambda, \mathrm{A}$ & $\mathrm{IEL}$ \\
\hline Cardelli et al. (1989) & $0.3-1.1(\mathrm{IR})$ & $9100-33,000$ & $A_{\lambda} / A_{V}$ \\
& $1.1-3.3(\mathrm{Opt} / \mathrm{NIR})$ & $3000-9100$ & \\
& $3.3-8(\mathrm{UV})$ & $1250-3000$ & \\
& $8-10(\mathrm{Far}-\mathrm{UV})$ & $1000-1250$ & \\
\hline O’Donnell (1994) & $1.1-3.3(\mathrm{Opt} / \mathrm{NIR})$ & $3000-9100$ & $A_{\lambda} / A_{V}$ \\
\hline Fluks et al. (1994) & $0-10(\mathrm{IR} / \mathrm{Opt} / \mathrm{UV})$ & $1000-2,500,000$ & $A_{\lambda} / E_{B-V}$ \\
\hline Larson \& Whittet (2005) & $0-0.8(\mathrm{IR})$ & $>12,500$ & $A_{\lambda} / A_{V}$ \\
\hline Fitzpatrick \& Massa (2007) & $0-1$ (IR) & $>10,000$ & $E_{\lambda-V} / E_{B-V}$ \\
& $3.7-8.7(\mathrm{UV})$ & $1150-2700$ & \\
\hline Gordon et al. (2009) & $3.3-11(\mathrm{UV} /$ Far-UV) & $900-3000$ & $A_{\lambda} / A_{V}$ \\
\hline
\end{tabular}

To properly obtain astrophysical parameters from catalogued photometry one also needs to study the possibility and sphere of application of the parameterization method. We indicate areas in the parameter space [effective temperature $\log T_{\text {eff }}$, gravity $\log g$, metallicity $\left[\mathrm{Fe} / \mathrm{H}\right.$ ], visual extinction $A_{V}$, total-to-selective extinction ratio $R_{V}$ ], where observational photometry precision, achieved in modern large multi-color surveys, allows us to obtain astrophysical parameters with acceptable accuracy Sichevskij et al. (2014).

\section{Determination of Interstellar Extinction}

Using the method pf parameterization, described in Section 3, one can construct $A_{V}(\mathrm{l}, \mathrm{b}, \mathrm{d})$ relation. This method allows one to plot parameterized objects in the distance-extinction $\left(d-A_{V}\right)$ plane, approximate them (by the cosecant law or more complicated function) and estimate interstellar extinction 
parameters in a given direction on the sky.

Note that for high galactic latitude areas $\left(|b|>15^{\circ}\right.$ or so) the interstellar extinction is thought to be (roughly) uniformly distributed and to satisfy the so-called cosecant (barometric) law, suggested by Parenago (1940). That function should be modified (complicated) for lower latitudes, as dust clouds concentrated in the Galactic plane, will have to be taken into account.

A preliminary analysis of applicability of SDSS and 2MASS photometry for determining the properties of stars and interstellar extinction was made by in Sichevskij (2018).

Then to test the procedure, described in Section 3, we have selected sky areas which are interesting from astrophysical point of view and where our results can be compared with independent studies. In particular, it is instructive and useful to apply the model to estimate interstellar extinction for several areas of the sky where individual estimates were made by Schlegel et al. (1998), and used to calculate extinction for SNs in the Universe accelerating expansion study Perlmutter \& et al (1999).

In our study Malkov et al. (2018b) we have cross-matched objects in 2MASS, SDSS, GALEX and UKIDSS surveys in selected areas at high galactic latitudes, using Virtual Observatory facilities. As a result of the cross-matching, we obtained multi-wavelength ( $\mathrm{i}=9$ to 13 bands) photometric data for each object. We have applied the method, described in Section 3, to parameterize the stars and to construct $A_{V}(\mathrm{l}, \mathrm{b}, \mathrm{d})$ relations for selected areas in the sky.

We have compared our results with LAMOST Luo \& et al (2015) data and extinction values to distant SNs (based on IRAS and DIRBE microwave data), available in the literature. The comparison exhibits a good agreement (see Malkov et al. (2018b) for details). A comparison of our results with recently released Gaia DR2 data also demonstrates a good agreement for stars as faint as $19^{m} .6$ gSDSS, and shows that our method allows us to determine spectral type, distance and interstellar extinction of objects out to $4.5 \mathrm{kpc}$ Malkov et al. (2018a). It indicates that the proposed algorithm (after some modifications, required for low galactic latitudes) can be used for construction of a 3D map of interstellar extinction in the Milky Way Galaxy.

\section{Conclusions}

The study of the stellar physical properties as well as the spatial distribution of interstellar extinction, is important for many investigations of galactic and extragalactic objects. We have developed a method for determination of stellar parameters and interstellar extinctions from multicolor photometry. This method was applied to objects drawn from modern large photometric surveys and, in this work, we give a review of the surveys and discuss problems of cross-identification.

Our procedure may be modified to use the astrometric and spectral information on the studied objects as input parameters. In particular, our procedure can be modified to determine stellar parameters and interstellar extinction values from not only multicolor photometry but also using additional information such as precise parallaxes and spectral classification, where available, thus reducing the number of unknowns in Eq. (2). One notable improvement has come with the recent release of the Gaia DR2 set of parallaxes, which allows us to use distance as an input (rather than as a free) parameter. It should significantly increase the accuracy of our results, especially when we can substitute the more precise parallaxes from Gaia DR3 for the DR2 data we currently use. Our procedure can also be modified for stars with spectral classification available from LAMOST Luo \& et al (2015), the largest source of spectral classification of objects in the northern sky. LAMOST Data Release 4 contains data on $7.6 \times 10^{6}$ objects and is available through VizieR database $(\mathrm{V} / 153)$.

Also, our experience is thought to be a practical guide to issues that will be particular important as soon as the new surveys become available (LSST Ivezić et al. (2019), SAGE Zheng et al. (2018), UVIT Tandon \& et al (2017)).

\section{Acknowledgements}

The work was partly supported by the Russian Foundation for Basic Researches (project 19-07-01198), by NSFC/RFBR grant 20-52-53009, and by DST grant INT/RUS/RFBR/P-265 to JM. OM thanks the CAS President's International Fellowship Initiative (PIFI). SK was supported by European Structural and Investment Fund and the Czech Ministry of Education, Youth and Sports (Project CoGraDS - CZ.02.1.01/0.0/0.0/15 003/0000437). The work is partially performed according to the Russian Government Program of Competitive Growth of Kazan Federal University. This research has made use of NASA's Astrophysics Data System, 
Stellar and interstellar parameters from large photometric surveys

and use of the VizieR catalogue access tool, CDS, Strasbourg, France. This research made use of TOPCAT, an interactive graphical viewer and editor for tabular data (Taylor, 2005). The acknowledgements were compiled using the Astronomy Acknowledgement Generator.

\section{References}

Alam S., et al., 2015, Astrophys. J. Suppl. Ser. , 219, 12

Bailer-Jones C. A. L., Rybizki J., Fouesneau M., Mantelet G., Andrae R., 2018, Astron. J. , 156, 58

Barentsen G., et al 2014, VizieR Online Data Catalog, 2321

Bianchi L., Herald J., Efremova B., Girardi L., Zabot A., Marigo P., Conti A., Shiao B., 2011, Astrophys. Space. Sci. , 335, 161

Bianchi L., Shiao B., Thilker D., 2017, Astrophys. J. Suppl. Ser. , 230, 24

Bono G., et al., 2019, Astrophys. J. , 870, 115

Cardelli J. A., Clayton G. C., Mathis J. S., 1989, Astrophys. J. , 345, 245

Castelli F., Kurucz R. L., 2003, in Piskunov N., Weiss W. W., Gray D. F., eds, IAU Symposium Vol. 210, Modelling of Stellar Atmospheres. p. A20 (arXiv:astro-ph/0405087)

Chambers K. C., et al 2016, arXiv e-prints,

Cirasuolo M., MOONS Consortium 2016, in Skillen I., Balcells M., Trager S., eds, Astronomical Society of the Pacific Conference Series Vol. 507, Multi-Object Spectroscopy in the Next Decade: Big Questions, Large Surveys, and Wide Fields. p. 109

Cutri R. M., et al 2003, VizieR Online Data Catalog, 2246

Cutri R. M., et al 2014, VizieR Online Data Catalog, 2328

DENIS Consortium 2005, VizieR Online Data Catalog, 1

Draine B. T., 2003, Ann. Rev. Astron. Astrophys. , 41, 241

Drew J. E., et al., 2014, Mon. Not. R. Astron. Soc. , 440, 2036

Eker Z., et al., 2018, Mon. Not. R. Astron. Soc. , 479, 5491

Falcón-Barroso J., Sánchez-Blázquez P., Vazdekis A., Ricciardelli E., Cardiel N., Cenarro A. J., Gorgas J., Peletier R. F., 2011, Astron. Astrophys. , 532, A95

Findeisen K., Hillenbrand L., Soderblom D., 2011, Astron. J. , 142, 23

Fitzpatrick E. L., Massa D., 2007, Astrophys. J. , 663, 320

Fluks M. A., Plez B., The P. S., de Winter D., Westerlund B. E., Steenman H. C., 1994, Astron. and Astrophys. Suppl. Ser. , 105, 311

Gaia Collaboration et al., 2018, Astron. Astrophys. , 616, A1

Gordon K. D., Cartledge S., Clayton G. C., 2009, Astrophys. J. , 705, 1320

Green G. M., et al 2015, Astrophys. J. , 810, 25

Gustafsson B., Edvardsson B., Eriksson K., Jørgensen U. G., Nordlund Å., Plez B., 2008, Astron. Astrophys. , 486, 951

Hanson R. J., Bailer-Jones C. A. L., 2014, Mon. Not. R. Astron. Soc. , 438, 2938

Indebetouw R., et al., 2005, Astrophys. J. , 619, 931

Ivezić Ž., et al., 2019, Astrophys. J. , 873, 111

Karpov S. V., Malkov O. Y., Mironov A. V., 2012, Astrophysical Bulletin, 67, 82

Kilpio E. Y., Malkov O. Y., Mironov A. V., 2012, in Prugniel P., Singh H. P., eds, Astronomical Society of India Conference Series Vol. 6, Astronomical Society of India Conference Series. p. 31 (arXiv:1208.1960)

Kraus A. L., Hillenbrand L. A., 2007, Astron. J. , 134, 2340

Kunder A., et al 2017, Astron. J. , 153, 75

Lallement R., et al., 2018, Astron. Astrophys. , 616, A132

Larson K. A., Whittet D. C. B., 2005, Astrophys. J. , 623, 897

Lawrence A., et al 2007, Mon. Not. R. Astron. Soc. , 379, 1599

Malkov O. et al. 
Le Borgne J.-F., et al., 2003, Astron. Astrophys. , 402, 433

Lejeune T., Cuisinier F., Buser R., 1997, Astron. and Astrophys. Suppl. Ser. , 125, 229

Luo A.-L., et al 2015, Research in Astronomy and Astrophysics, 15, 1095

Luo A. L., Zhao Y. H., Zhao G., et al. 2019, VizieR Online Data Catalog, p. V/164

Malkov O., Karpov S., 2011, in Evans I. N., Accomazzi A., Mink D. J., Rots A. H., eds, Astronomical Society of the Pacific Conference Series Vol. 442, Astronomical Data Analysis Software and Systems XX. p. 583

Malkov O., Kilpio E., 2002, Astrophys. Space. Sci. , 280, 115

Malkov O. Y., Sichevskij S. G., Kovaleva D. A., 2010, Mon. Not. R. Astron. Soc. , 401, 695

Malkov O., Mironov A., Sichevskij S., 2011, Astrophys. Space. Sci. , 335, 105

Malkov O., Dluzhnevskaya O., Karpov S., Kilpio E., Kniazev A., Mironov A., Sichevskij S., 2012, Baltic Astronomy, 21,319

Malkov O., et al., 2018a, Galaxies, 7, 7

Malkov O., et al., 2018b, Open Astronomy, 27, 62

Malkov O. Y., et al., 2019, CEUR Workshop Proceedings, 2523, 217

Malkov O., Kovaleva D., Sichevsky S., Zhao G., 2020, Research in Astronomy and Astrophysics, 20, 139

O’Donnell J. E., 1994, Astrophys. J. , 437, 262

Ochsenbein F., et al., 2004, in Quinn P. J., Górski K. M., eds, Toward an International Virtual Observatory. p. 118, doi: $10.1007 / 10857598^{\circ} 18$

Parenago P. P., 1940, Astron. Zh., 13, 3

Pecaut M. J., Mamajek E. E., 2013, Astrophys. J. Suppl. Ser. , 208, 9

Perlmutter S., et al 1999, Astrophys. J. , 517, 565

Pickles A. J., 1998, Publ. Astron. Soc. Pac. , 110, 863

Prugniel P., Soubiran C., Koleva M., Le Borgne D., 2007, arXiv Astrophysics e-prints,

Reis I., Poznanski D., Baron D., Zasowski G., Shahaf S., 2018, Mon. Not. R. Astron. Soc. , 476, 2117

Rieke G. H., Lebofsky M. J., 1985, Astrophys. J. , 288, 618

Sale S. E., et al., 2014, Mon. Not. R. Astron. Soc. , 443, 2907

Samus' N. N., Kazarovets E. V., Durlevich O. V., Kireeva N. N., Pastukhova E. N., 2017, Astronomy Reports, 61, 80

Schlafly E. F., Finkbeiner D. P., 2011, Astrophys. J. , 737, 103

Schlegel D. J., Finkbeiner D. P., Davis M., 1998, Astrophys. J. , 500, 525

Sichevskij S. G., 2018, Astrophysical Bulletin, 73, 98

Sichevskij S. G., Mironov A. V., Malkov O. Y., 2014, Astrophysical Bulletin, 69, 160

Sichevskiy S. G., Mironov A. V., Malkov O. Y., 2013, Astronomische Nachrichten, 334, 832

Sichevsky S., Malkov O., 2016, Baltic Astronomy, 25, 67

Skvortsov N. A., et al., 2016, Astrophysical Bulletin, 71, 114

Smith D. J. B., et al 2016, in Reylé C., Richard J., Cambrésy L., Deleuil M., Pécontal E., Tresse L., Vauglin I., eds, SF2A-2016: Proceedings of the Annual meeting of the French Society of Astronomy and Astrophysics. pp 271-280 (arXiv:1611.02706)

Straižys V., 1992, Multicolor stellar photometry. Tucson : Pachart Pub. House, c1992.

Tandon S. N., et al 2017, Astron. J. , 154, 128

Taylor M. B., 2005, in Shopbell P., Britton M., Ebert R., eds, Astronomical Society of the Pacific Conference Series Vol. 347, Astronomical Data Analysis Software and Systems XIV. p. 29

Valdes F., Gupta R., Rose J. A., Singh H. P., Bell D. J., 2004, Astrophys. J. Suppl. Ser. , 152,251

Wu Y., Singh H. P., Prugniel P., Gupta R., Koleva M., 2011, Astron. Astrophys. , 525, A71

Yanny B., et al 2009, Astron. J. , 137, 4377

Yuan H. B., Liu X. W., Xiang M. S., 2013, Mon. Not. R. Astron. Soc. , 430, 2188

Zheng J., Zhao G., Wang W., Fan Z., Tan K.-F., Li C., Zuo F., 2018, Research in Astronomy and Astrophysics, 18,147

de Jong R. S., et al., 2019, The Messenger, 175, 3

Malkov O. et al. 